\title{
Österreichische Nationalbibliothek
}

\author{
Fritze,Christiane; christiane.fritze@onb.ac.at / Steindl,Christoph; christoph.steindl@onb.ac.at
}

An der Österreichischen Nationalbibliothek (ÖNB), einer der bedeutendsten Gedächtnisinstitutionen des Landes, wird eine übergreifende, nachhaltige Infrastruktur für Digitale Editionen aufgebaut. Alle künftigen Digitalen Editionen, die auf Quellenmaterial der Österreichischen Nationalbibliothek basieren, werden darin realisiert. Die Infrastruktur wird als Teil der Digital HumanitiesStrategie zur Erfüllung der Vision 2025 der Österreichischen Nationalbibliothek entwickelt.

Die Editionsinfrastruktur verbindet Komponenten zur Erstellung von Digitalen Editionen mit der Repository-Komponente zur Bereitstellung der onlinePräsentation der Edition. Dabei wurde ein modularer Aufbau gewählt, sodass die Basiskonfiguration, die für jede Edition bereitgestellt wird, projektspezifisch adaptiert werden kann.

Bei der Erstellungskomponente kommt der oXygen-XML-Editor zum Einsatz, in dem eine ausgearbeitete Projektstruktur aufgesetzt wird. Das Quellmaterial wird als TEI-XML aufbereitet und in einem GitLab-Repository für die Bearbeitung des Editionsteams versioniert abgelegt. Den XML-Dateien liegt ein an der ÖNB entwickeltes Basisschema zugrunde, das mit projektspezifischen Elementen erweitert werden kann.

Technische Grundlage der Repository-Komponente bildet eine eigenständige Instanz des Geisteswissenschaftlichen Asset Management Systems (GAMS), das am Zentrum für Informationsmodellierung - Austrian Centre for Digital Humanities der Karl-Franzens-Universität Graz entwickelt wurde. Das edierte Quellmaterial in XML wird via XSLT-Transformationen zur Anzeige gebracht. Digitalisate an der Österreichischen Nationalbibliothek werden sowohl inhouse als auch mit externen Partnerunternehmen erstellt und in der Regel via IIIF zur Verfügung gestellt und so in die Digitale Edition eingebunden.

Das Design für die Editionsprojekte wurde dahingehend entwickelt, dass es für neue Editionsprojekte wiederverwendet werden kann. Als Unterscheidungsmerkmal zwischen den Digitalen Editionen dient hier das Farbschema, das von einer Primär- und einer Sekundärfarbe geprägt ist. Das Design wurde mit Bootstrap umgesetzt und ist auch für mobile Endgeräte bzw. Geräte mit kleinerem Display aufbereitet.

\section{Literatur:}

- Fritze, Christiane: Wohin mit der digitalen Edition? Ein Beitrag aus der Perspektive der Österreichischen Nationalbibliothek Wohin mit der digi- 
talen Edition?. In: Bibliothek - Forschung und Praxis 43 (3): 2019, S. $432-440$.

- Digitale Editionen an der Österreichischen Nationalbibliothek. URL: https: //edition.onb.ac.at/start/o:ode.home/methods/sdef :TEI/get

- Fritze, Christiane; Steindl, Christoph: Nachhaltige Infrastruktur für digitale Editionen an der Österreichischen Nationalbibliothek. Berlin: 2018, URL: urn:nbn:de:0290-opus4-35161.

\section{Software:}

Bootstrap, GAMS, Gitlab, iiif, Oxygen

\section{Verweise:}

Digitale Edition, GAMS, International Image Interoperability Framework, TEI

\section{Projekte:}

Tagebücher Andreas Okopenko, Briefwechsel August Sauer Bernhard Seuffert 1880 bis 1926 digital, Blotius Digital. Digitale Edition des Inventars der Wiener Hofbibliothek (1576), Infrastruktur für Digitale Editionen an der Österreichischen Nationalbibliothek, Vision 2025 der Osterreichischen Nationalbibliothek

\section{Themen:}

Institutionen, Software und Softwareentwicklung

\section{Zitiervorschlag:}

Fritze, Christiane; Steindl, Christoph. 2021. Österreichische Nationalbibliothek. In: KONDE Weißbuch. Hrsg. v. Helmut W. Klug unter Mitarbeit von Selina Galka und Elisabeth Steiner im HRSM Projekt "Kompetenznetzwerk Digitale Edition". URL: https://gams.uni-graz.at/o:konde.153 